\title{
OPEN An Internally Quenched Fluorescent Peptide Substrate for Protealysin
}

\author{
Maria A. Karaseva ${ }^{1}$, Ksenia N. Chukhontseva ${ }^{1}$, Irina S. Lemeskina ${ }^{1}$, Marina L. Pridatchenko ${ }^{2}$, \\ Sergey V. Kostrov ${ }^{1} \&$ llya V. Demidyuk $\mathbb{B D}^{1}$
}

Received: 12 May 2019

Protealysin, a metalloprotease of Serratia proteamaculans, is the prototype of a subgroup of the M4 peptidase family. Protealysin-like proteases (PLPs) are widely spread in bacteria but also occur in fungi and certain archaea. The interest in PLPs is primarily due to their putative involvement in the bacterial pathogenesis in animals and plants. Studying PLPs requires an efficient quantitative assay for their activity; however, no such assay has been reported so far. Here, we used the autoprocessing site sequence of the protealysin precursor to construct an internally quenched fluorescent peptide substrate

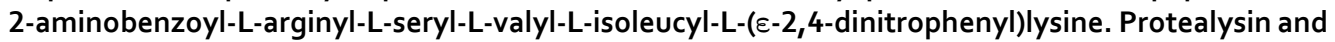
thermolysin, the prototype of the M4 family, proved to hydrolyze only the Ser-Val bond of the substrate. The substrate exhibited a $\mathrm{K}_{\mathrm{M}}=35 \pm 4 \mu \mathrm{M}$ and $\mathrm{k}_{\text {cat }}=21 \pm 1 \mathrm{~s}^{-1}$ for protealysin as well as a $\mathrm{K}_{\mathrm{M}}=33 \pm 8 \mu \mathrm{M}$ and $k_{\text {cat }}=7 \pm 1 \mathrm{~s}^{-1}$ for thermolysin at $37^{\circ} \mathrm{C}$. Comparison of the effect of different enzymes (thermolysin, trypsin, chymotrypsin, savinase, and pronase E) on the substrate has demonstrated that it is not strictly specific for protealysin; however, this enzyme has higher molar activity even compared to the closely related thermolysin. Thus, the proposed substrate can be advantageous for quantitative studies of protealysin as well as for activity assays of other M4 peptidases.

Protealysin (PLN), a metalloprotease of Serratia proteamaculans, is the prototype of a subgroup of the M4 peptidase family (https://www.ebi.ac.uk/merops/cgi-bin/famsum?family=M4) ${ }^{1}$. Protealysin-like proteases (PLPs) are widely spread in bacteria but also occur in fungi and certain archaea. The interest in PLPs is primarily due to their probable involvement in the bacterial pathogenesis in animals and plants. For instance, PLN and grimelysin of Serratia grimesii ${ }^{2}$ were shown to mediate bacterial invasion of eukaryotic cells ${ }^{3-6}$; protease Zpx of Cronobacter turicensis, a causative agent of life-threatening systemic infections in premature, low-birth-weight, and immune-compromised neonates is presumably a component of the bacterial mechanism of immune evasion $^{7}$; protease PrtS of the insect-pathogenic bacterium Photorhabdus luminescens can suppress the antibacterial defense in insects ${ }^{8,9}$; while protease Prt1 of the plant-pathogenic bacterium Pectobacterium carotovorum affects extensins, plant cell wall protein, which makes Prt1 a putative factor of bacterial penetration into plant cells ${ }^{10}$.

Currently, the activity of PLPs is assayed using protein substrates, most commonly, casein (typically azocasein $)^{8-16}$ or less commonly actin ${ }^{3,15,17}$. The applicability of these substrates is somewhat limited. In the case of casein, numerous bonds are cleaved and the enzymatic reaction is evaluated by the products not precipitated by trichloroacetic acid. Thus, the degree of substrate conversion cannot be correctly evaluated. In addition, the kinetics monitoring interferes with the precipitation-based end point assay. Actin is specifically digested by $\mathrm{PLPs}^{3}$. However, the routine analysis relies on the fractionation of the hydrolytic products by gel electrophoresis, which makes it time- and labor-consuming. A well-known substrate of neutral proteases N-(3-[2-Furyl]acryloyl)-Gly-Leu amide (FAGLA) ${ }^{18}$ was tested. However, PLN proved to hydrolyze FAGLA with very low efficiency $\left(\mathrm{k}_{\text {cat }} / \mathrm{K}_{\mathrm{M}} \text { is roughly } 100 \text { times lower compared to thermolysin, a member of the same } \mathrm{M} 4 \text { family of peptidases }\right)^{12}$, which makes it impracticable for PLP activity assay.

The problem of a convenient protease substrate can be successfully obviated by using reporter systems based on Förster resonance energy transfer (FRET). FRET substrates of proteases are peptides corresponding to the enzyme specificity with a donor fluorophore at one end and a fluorescence acceptor at the other. The acceptor can be "dark" and function as a quencher or fluoresce. In addition to the flexibility, FRET substrates are advantageous in high sensitivity of the corresponding methods and the possibility of the continuous kinetic assay. FRET

${ }^{1}$ Institute of Molecular Genetics, Russian Academy of Sciences, Moscow, Russia. ${ }^{2}$ V.L. Talrose Institute for Energy Problems of Chemical Physics, N.N. Semenov Federal Research Center of Chemical Physics, Russian Academy of Sciences, Moscow, Russia. Correspondence and requests for materials should be addressed to I.V.D. (email: duk@ img.ras.ru) 


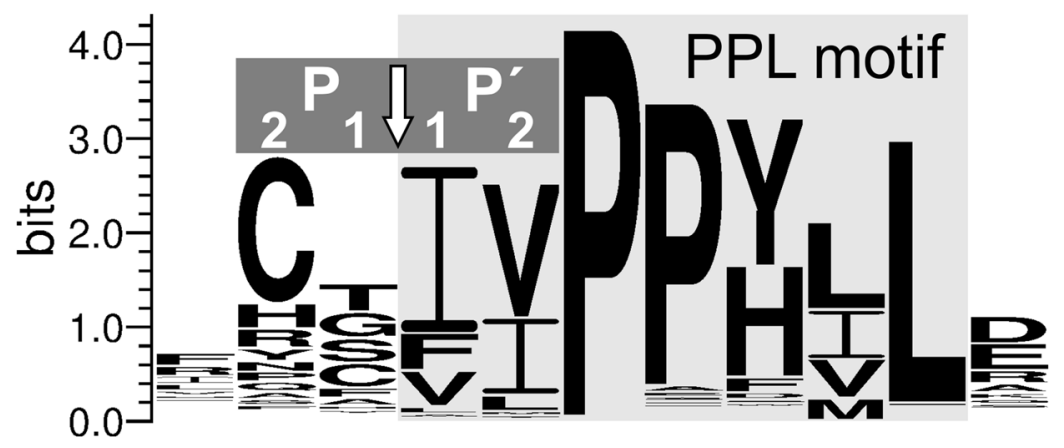

Figure 1. LOGO presentation of processing site and PPL motif in precursors of protealysin-like proteases. The arrow indicates the cleavage site. The processing site residues are designated as $\mathrm{P} 1, \mathrm{P} 2, \mathrm{P} 1^{\prime}, \mathrm{P} 2^{\prime}$ according to Schechter and Berger ${ }^{26}$. Light gray shading indicates the PPL motif.

substrates are used in the activity assays for a variety of proteases including certain M4 peptidases ${ }^{19-25}$. However, no FRET substrates have been created for PLPs.

Here, the sequence of the autoprocessing site of the PLN precursor was used to construct an internally quenched fluorescent peptide substrate. The substrate examination has demonstrated that it can be efficiently used for quantitative analysis of PLN catalytic properties and can be also used to assay the activity of other M4 peptidases.

\section{Results and Discussion}

Substrate design. The substrate specificity of PLPs has not been studied systematically ${ }^{1}$. At the same time, the analysis of the PLN spatial structure in comparison with that of thermolysin (TLN) indicates that, similar to other M4 peptidases, the $\mathrm{S}^{\prime}$ site (as designated by Schechter and Berger ${ }^{26}$ ) is the major determinant of the substrate specificity of PLPs ${ }^{27}$. However, the interactions at the S2' site of PLN were proposed to modulate the efficiency of substrate binding more than for TLN. The interactions of the substrate downstream of the scissile bond are determined by the S2 and S1 sites of the enzyme and are largely analogous to those of TLN ${ }^{27}$. Thus, efficient $\mathrm{PLN}$ binding requires four substrate sites (P2, $\mathrm{P} 1, \mathrm{P} 1^{\prime}$, and $\left.\mathrm{P} 2^{\prime}\right)$. The hydrolysis sites have been determined for several proteins by certain PLPs (PLN, grimelysin, and Prt1). These data indicate the preference of the enzymes for hydrophobic residues at the $\mathrm{P} 1^{\prime}$ and $\mathrm{P} 2^{\prime}$ positions, which was more pronounced for the residues with less voluminous side chains compared to TLN. The preference for the P1 and P2 positions was less pronounced; however, a bias to small and polar residues at P1 and charged ones at P2 was observed ${ }^{1,3,10,17,28,29}$. The substrate sequence was selected based on the autocatalytic maturation data for PLP precursors. It was shown that PLN is accumulated in the cell as an inactive precursor, which is processed and activated after cell lysis ${ }^{29,30}$. PLN contributes most to the processing. It goes step-by-step; and the first autoprocessing event, the detachment of seven N-terminal residues, takes place within the cell ${ }^{29}$, and the processing site is located directly upstream of the PPL-motif conserved for propeptides of PLPs (Fig. 1) ${ }^{1,31}$. The substrate design was based on the PLN processing site Arg-Ser-|-Val-Ile (" $-\mid-$ " is the cleaved bond). At the same time, the residues at the $\mathrm{P}^{\prime}$ and $\mathrm{P} 2^{\prime}$ positions of the processing site in PLN are common in other enzymes of the group, suggesting that such substrate can be applied for the activity assay of all PLPs disregarding their nuances in substrate specificity.

Next, we selected the donor fluorophore and fluorescence acceptor. Currently, a variety of donor-acceptor pairs are used in protease substrates ${ }^{23,25,32,33}$. They vary in the structure, spectral and other physicochemical properties, and not least in price. We opted for the long and widely used pair 2-aminobenzoyl/2,4-dinitrophenyl (Abz/ Dnp). This pair has a clear drawback relative to newer products, high background fluorescence when used as a part of internally quenched fluorescent substrates ${ }^{33}$. This results in a relatively low sensitivity of activity assays based on Abz/Dnp peptides; however, it is not critical as demonstrated by numerous examples of their successful application to address standard problems ${ }^{32}$. At the same time, the Abz/Dnp pair has clear merits ${ }^{34}$. Abz is the smallest fluorescent group described so far, which ensures the solubility of Abz-containing peptides. Dnp is a stable quencher and its absorption spectrum does not change with $\mathrm{pH}$, which makes this group a convenient marker for substrate quantitation in solutions. Finally, Abz and Dnp are inexpensive, can be readily integrated into peptide substrates, and are among standard modifications provided by most custom peptide synthesis services.

Abz was attached to the $\mathrm{N}$ terminus of the peptide. Dnp was introduced into the peptide by linking to the $\varepsilon$-amino group of lysine added to the $C$ terminus of the peptide. Thus, the substrate proposed in this study has the following structure: Abz-Arg-Ser-Val-Ile-Lys(Dnp) (Abz-RSVIK(Dnp)).

Substrate absorption and solubility. Abz-RSVIK(Dnp) features sorption onto quartz cuvettes for spectrophotometry, microplates for fluorescence-based assays, and microcentrifuge tubes including low protein-binding tubes (supplementary Fig. S1), which has to be accounted for when using this substrate. First, we excluded the common serial dilutions and prepared all working solutions directly from the substrate stock in DMSO. Second, substrate solutions were incubated in plate wells prior to enzymatic assays, which obviated concentration changes during the experiment (Supplementary Fig. S2). Third, the concentration alterations from absorption were controlled in a parallel control experiment without the substrate for each activity assay to evaluate the substrate concentration directly in the reaction mixture. (All details of the assay for the initial hydrolysis 

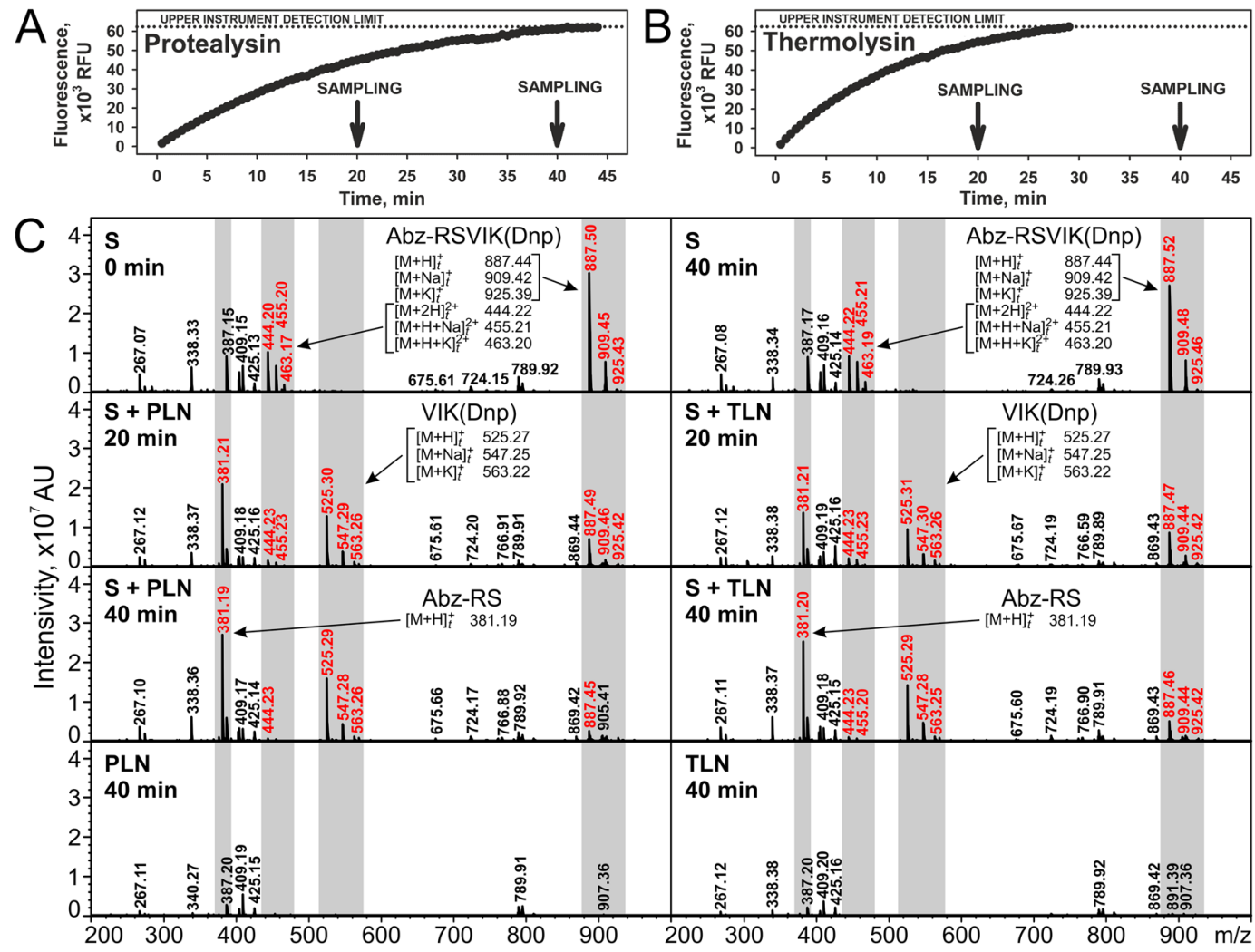

Figure 2. Analysis of Abz-RSVIK(Dnp) cleavage by protealysin (PLN) and thermolysin (TLN). (A,B) Kinetics of Abz-RSVIK(Dnp) hydrolysis by PLN and TLN, respectively. The arrows indicate time points of mass spectrometry. RFU, relative fluorescence units. (C) Mass-spectrometric analysis of reaction mixes. The peaks corresponding to the substrate and hydrolysis products are indicated by red font and gray vertical bars. $\mathrm{S}$, substrate; $\mathrm{AU}$, arbitrary units; $\mathrm{m} / \mathrm{z}$, mass-to-charge ratio; $[\mathrm{M}+\mathrm{nH} / \mathrm{K} / \mathrm{Na}(+\mathrm{Na} / \mathrm{K})]^{\mathrm{Z}+}{ }_{\mathrm{t}}$, theoretical $\mathrm{m} / \mathrm{z}$ values.

rate are described in Methods.) As concerns the sorption onto spectrophotometer cuvettes, it is relatively low (Supplementary Fig. S1A), and considering that obviating it is hardly realistic, we tried to perform the measurements as fast as possible. In our experiments, the optical density was measured $10-15 \mathrm{~s}$ after solutions were added to cells.

The substrate sorption on labware complicated the measurement of the Abz-RSVIK(Dnp) solubility since the standard approach based on serial dilutions and identification of the linear range of the experimental concentration-dilution factor relationship could not be used. The substrate solubility was evaluated by periodic spectrophotometric quantitation of its dilutions immediately prepared from the stock in DMSO for one day. Up to $130 \mu \mathrm{M}$, the substrate solutions remained stable for $24 \mathrm{~h}$ (Supplementary Fig. S3); however, starting from $150 \mu \mathrm{M}$ their optical density decreased with time. Thus, the substrate has reasonable water solubility and can be used in concentrations up to $130 \mu \mathrm{M}$.

Identification of substrate hydrolysis site by protealysin and thermolysin. Mass spectrometry of the reaction mixtures was used to identify the site of Abz-RSVIK(Dnp) hydrolysis by PLN and TLN. This analysis detected only the products corresponding to hydrolysis at the Ser-Val bond, which is also cleaved in the natural amino acid sequence reproduced in the synthetic peptide (Fig. 2). The specificity of the enzymes also includes cleavage of the bond preceding Ile suggesting the Val-Ile digestion of the substrate. However, even if such reaction takes place it proceeds substantially slower and its products remain undetectable after the time period ( $40 \mathrm{~min})$ by far exceeding that required for almost complete peptide hydrolysis at Ser-Val. In the context of Abz-RSVIK(Dnp) application in the activity assays, it is fair to say that its hydrolysis occurs at the only bond. Thus, the quantity of the cleavage product detected by its fluorescence is equivalent to the quantity of hydrolyzed substrate, which allows adequate quantitation of the enzyme kinetics.

Kinetic parameters for substrate hydrolysis by protealysin and thermolysin. The dependence of the initial rates of Abz-RSVIK(Dnp) hydrolysis by PLN and TLN on the substrate concentration fits the Michaelis-Menten equation (Fig. 3). According to the kinetic parameters, the efficiency $\left(\mathrm{k}_{\mathrm{cat}} / \mathrm{K}_{\mathrm{M}}\right)$ of substrate hydrolysis by PLN is roughly three times higher (Table 1), which can be attributed to the changes in $\mathrm{k}_{\text {cat }}$ rather than in $\mathrm{K}_{\mathrm{M}}$. Thus, the substrate binds equally well to both enzymes, and the decreased efficiency of TLN can be due to inaccurate positioning of the bond to be digested relative to the catalytic site as a result of too deep entrance of the small lateral radical at $\mathrm{P}^{\prime}$ of the substrate's Val into the deep $\mathrm{S1}^{\prime}$ pocket of $\mathrm{TLN}^{27}$. 


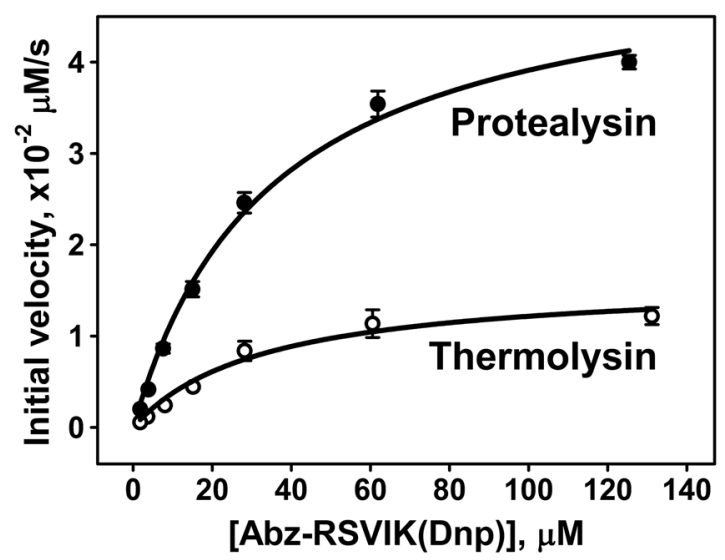

Figure 3. The dependence of the initial rates of Abz-RSVIK(Dnp) hydrolysis by protealysin and thermolysin on the substrate concentration. The enzymes concentration was $2.5 \mathrm{nM}$. Values are represented as mean $\pm \mathrm{SD}$ of two independent experiments with triplicates. Solid lines, fit of the data to the Michaelis-Menten equation $\left(\mathrm{R}^{2}=0.9939\right.$ for protealysin and $\mathrm{R}^{2}=0.9755$ for thermolysin).

\begin{tabular}{|l|l|l|l|}
\hline Enzyme & $\mathbf{K}_{\mathbf{M}} \pm \mathbf{S E}, \boldsymbol{\mu M}$ & $\mathbf{k}_{\text {cat }} \pm \mathbf{S E}, \mathbf{s}^{-1}$ & $\mathbf{k}_{\text {cat }} / \mathbf{K}_{\mathbf{M}} \pm \mathbf{S E}, \mathbf{s}^{-\mathbf{1}} \boldsymbol{\mu \mathbf { M } ^ { - 1 }}$ \\
\hline Protealysin & $35 \pm 4$ & $21 \pm 1$ & $0.60 \pm 0.07$ \\
\hline Thermolysin & $33 \pm 8$ & $7 \pm 1$ & $0.21 \pm 0.06$ \\
\hline PLN/TLN & 1.1 & 3.0 & 2.8 \\
\hline
\end{tabular}

Table 1. Kinetic parameters for hydrolysis of Abz-RSVIK(Dnp) by protealysin and thermolysin at $37^{\circ} \mathrm{C}$. SE, standard error; PLN, protealysin; TLN, thermolysin.

This result critically differs from the above-mentioned pattern of FAGLA hydrolysis where the efficiency of TLN is 100 that of $\mathrm{PLN}^{12}$. Poor efficiency of PLN as a substrate can be attributed to the Leu-P1' inconformity to the PLN's substrate specificity. However, the hydrolysis of protein substrates at sites preceding Leu has been demonstrated for PLN and its close homolog ECP32/grimelisin ${ }^{29,35}$, which suggests that amino acids at non-P1' positions are more significant for the PLN-substrate interaction. This agrees with the previously proposed hypothesis that the efficiency of substrate binding depends on the amino acid at the substrate $\mathrm{P} 2^{\prime}$ position more for PLN than for TLN based on the analysis of 3D structures ${ }^{27}$.

Thus, Abz-RSVIK(Dnp) is the first low-molecular-weight substrate efficiently hydrolyzed by PLN and can be used to study its catalytic properties. In addition, the substrate can be used to assay activities of other M4 peptidases.

Hydrolysis of the substrate by other enzymes. The amino acid sequence of Abz-RSVIK(Dnp) suggests that this substrate can be hydrolyzed by both M4 peptidases and other proteases. We tested this suggestion using several proteases whose specificity (according to published data) allows the cleavage of the proposed substrate. We used trypsin with the preference for peptide bonds next to Arg and Lys; chymotrypsin cleaving bonds next to Val, Ile, and Ser ${ }^{36}$; and savinase cleaving bonds next to Val, Ile, and $\mathrm{Arg}^{37}$ (however, these amino acids are not preferred at P1 for chymotrypsin and savinase); as well as pronase E, a mixture of Streptomyces griseus peptidases with different activity and specificity ${ }^{38}$.

This comparison demonstrates the highest efficiency of Abz-RSVIK(Dnp) hydrolysis by PLN among the tested enzymes. A parallel test using azocasein demonstrated a different pattern (Fig. 4). Despite its wide substrate specificity, PLN is not a leader here: it is inferior to TLN and pronase $\mathrm{E}$ and on par with savinase. The data obtained indicate that Abz-RSVIK(Dnp) is not a strictly specific substrate for PLN but its structure fits more the specificity of this metalloprotease compared to other tested enzymes (including the related TLN), which makes it acceptable considering the wide substrate specificity of PLN. At the same time, Abz-RSVIK(Dnp) is not specific enough to detect protealysin-like proteases in natural producers. This is indicated by our comparison of cell lysate activities in E. coli TG1 (pUC19) lacking M4 peptidase genes (according to the genome analysis) and the same strain transformed by the pSP1.8 plasmid obtained and described by us previously. This construct contains a fragment of S. proteamaculans DNA $(\sim 3 \mathrm{~kb})$ inserted into the BamHI site of pUC19. The cloned fragment contains the PLN gene with its own regulatory elements providing for its expression ${ }^{12}$. In the case of E. coli TG1 (pSP1.8) the activity was $\sim 100$ higher relative to E. coli TG1 (pUC19) (Fig. 5). However, the cloning vector used, pUC19, is a high-copy-number plasmid maintained at 500-700 copies per cell ${ }^{39}$. Thus, the PLN gene dose was by more than two orders of magnitude higher in our experiment than in the natural producer. Accordingly, one can expect low levels of this enzyme and sub-background levels of its activity in the natural producer. 


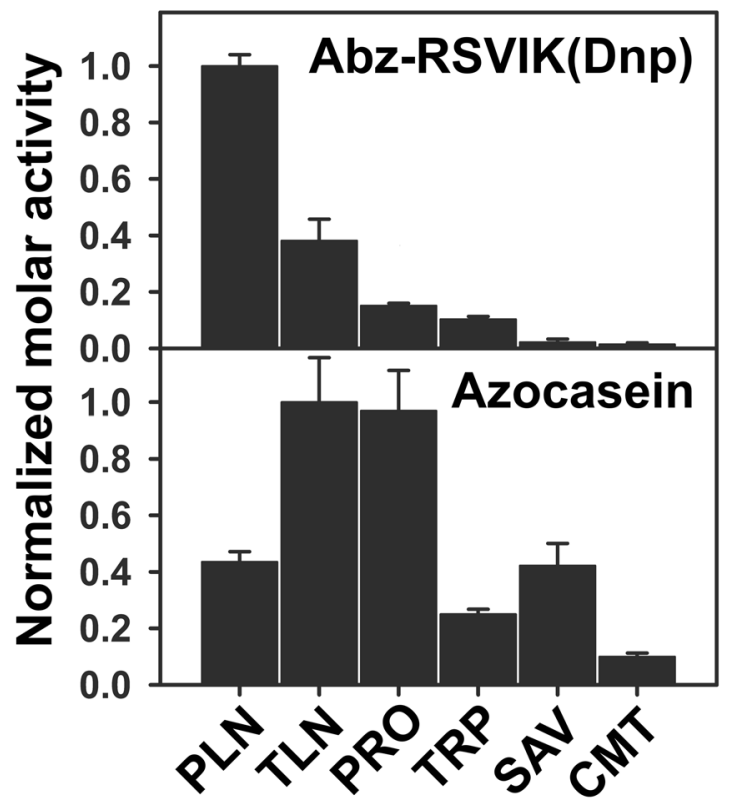

Figure 4. Hydrolysis of Abz-RSVI-K(Dnp) and azocasein by different enzymes. PLN, protealysin; TLN, thermolysin; PRO, pronase E; TRP, trypsin; SAV, savinase; and CMT, chymotrypsin. The activity was evaluated for $60 \mu \mathrm{M}$ Abz-RSVI-K(Dnp); $2.5 \mathrm{nM}$ PLN, TLN, TRP, SAV, and CMT; and $1.7 \mathrm{nM}$ PRO. Enzyme concentrations were determined after Bradford. The following molecular weights were used in the calculation: PLN, $31.9 \mathrm{kDa}$; TLN, $34.6 \mathrm{kDa}$; PRO, $40 \mathrm{kDa}$ (the mean molecular weight of the PRO enzymes); TRP, $23.8 \mathrm{kDa}$; SAV, 26.7 kDa; and CMT, $25 \mathrm{kDa}$. Values are represented as the mean and SD of three independent experiments.

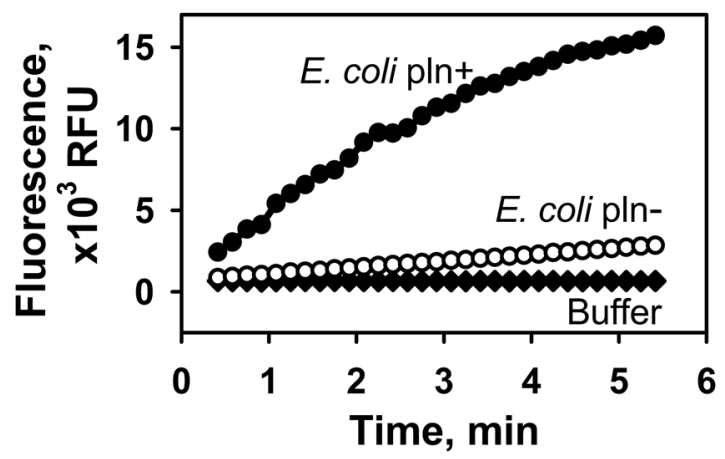

Figure 5. Effect of bacterial lysates on Abz-RSVI-K(Dnp). Abz-RSVI-K(Dnp) (40 $\mu \mathrm{M})$ was incubated with: $E$. coli pln+, lysate of E. coli TG1 cells transformed with pSP1.8 containing the locus of Serratia proteamaculans genomic DNA with the protealysin gene ${ }^{12}$; E. coli pln-, lysate of E. coli TG1 cells transformed with the empty cloning vector pUC19; Buffer, buffer without cell lysates. Lysates obtained from $5 \cdot 10^{4} \mathrm{E}$. coli cells were used for the activity assays. RFU, relative fluorescence units.

\section{Conclusions}

The first substrate applicable to quantitative studies of protealysin catalytic properties has been constructed. This substrate can also be used for activity assays of other M4 peptidases.

\section{Methods}

Materials. In this work we used tris-(hydroxymethyl)aminomethane (Tris) (Amresco, USA), ammonium bicarbonate, azocasein (Sigma, USA), dimethyl sulfoxide (DMSO) (MP Biomedicals, France), thermolysin (TLN) (Serva, Germany), pronase E (Serva, Germany), savinase (Novozymes, Denmark), trypsin (Spofa, Czech Republic), and chymotrypsin (Koch Light Laboratories, UK).

An internally quenched fluorescent peptide substrate for protealysin, 2-aminobenzoyl-L-arginylL-seryl-L-valyl-L-isoleucyl-L-( $\varepsilon$-2,4-dinitrophenyl)lysine (Abz-RSVIK(Dnp)), was synthesized by Peptide 2.0 (Chantilly, USA). The peptide purity was over $98 \%$ as determined by HPLC analysis. The results of the chromatography and mass-spectrometry analysis of Abz-RSVIK(Dnp) are given in the Supplementary Information File.

$\mathrm{His}_{6}$-tagged protealysin (PLN) was purified as described elsewhere ${ }^{29}$. Briefly, E. coli cells BL-21 (DE3) [pProPl$\mathrm{nHis}_{6}$ ] were grown with aeration at $37^{\circ} \mathrm{C}$ in Lennox $\mathrm{LB}^{40}$ containing $100 \mu \mathrm{g} / \mathrm{ml}$ ampicillin. PLN synthesis was 
induced by $0.5 \mathrm{mM}$ of isopropyl- $\beta$-D-thiogalactopyranoside (Sigma, USA) using standard protocol. Four h after induction, cells were collected by centrifugation, resuspended in $50 \mathrm{mM}$ Tris- $\mathrm{HCl}$ ( $\mathrm{pH} 8.0$ ), and ultrasonicated. After centrifugation, the supernatant was applied onto a Ni-NTA XPure Agarose Resin (UBPBio, USA) column equilibrated with the same buffer. PLN was eluted with $100 \mathrm{mM}$ imidazole. The fractions containing the target protein were concentrated on an Amicon Ultra-15 centrifugal filter unit (Millipore, USA) applied onto a Superdex 75 HR 10/30 column (GE Healthcare, Sweden) and eluted with the same buffer containing $0.15 \mathrm{M} \mathrm{NaCl}$. Finally, PLN was dialyzed against $50 \mathrm{mM} \mathrm{NH}_{4} \mathrm{HCO}_{3}$ and lyophilized.

All other reagents were of reagent grade and purchased from Dia-M (Russia).

Bioinformatics. Sequences of PLPs were extracted by protein BLAST (http://blast.ncbi.nlm.nih.gov) from the database of non-redundant protein sequences (nr) using the PLN precursor sequence (GenBank ID: AAV88082.1) as a query. Sequences whose identity to other sequences exceeded $85 \%$ were excluded using the CD-HIT web server (http://www.bioinformatics.org/cd-hit) ${ }^{41}$. The multiple sequence alignment of the remaining sequences was constructed by Clustal X2 (http://www.clustal.org/clustal2) ${ }^{42}$. The alignment was manually inspected to remove the sequences lacking the PPL motif. The resulting set included 821 sequences of PLPs. The LOGO presentation of the consensus sequence was generated by WebLogo 3.6 (http://weblogo.threeplusone. $\operatorname{com})^{43}$. The sequences used for LOGO generation are presented in the Supplementary Sequences File.

Protein and Abz-RSVIK(Dnp) concentrations. The concentrations of PLN and TLN were determined spectrophotometrically at $280 \mathrm{~nm}$ using the extinction coefficients $\varepsilon_{280 \mathrm{~nm}}=52370 \mathrm{M}^{-1} \mathrm{~cm}^{-1}$ and $\varepsilon_{280 \mathrm{~nm}}=58200 \mathrm{M}^{-1} \mathrm{~cm}^{-1}$, respectively. The extinction coefficients were calculated from the amino acid sequence using the ProtParam tool (http://www.expasy.org/tools/protparam.html).

The concentrations of other proteins were assayed after Bradford ${ }^{44}$ with modifications ${ }^{45}$ using $\operatorname{IgG}$ as a standard.

The Abz-RSVIK(Dnp) concentration was determined by the absorption of 2,4-dinitrophenyl at $365 \mathrm{~nm}$ using the extinction coefficient $\varepsilon_{365 \mathrm{~nm}}=17300 \mathrm{M}^{-1} \mathrm{~cm}^{-146}$. The optical density was recorded $10-15 \mathrm{~s}$ after the solution was introduced into the optical cell.

The optical density was registered using an Agilent 8453 UV-Vis Spectrophotometer.

Solubility of Abz-RSVIK(Dnp). The stock solutions $(6 \mu \mathrm{l})$ in DMSO were supplemented with $500 \mu \mathrm{l}$ of $50 \mathrm{mM}$ Tris- $\mathrm{HCl}(\mathrm{pH}$ 7.4). The solutions were incubated at room temperature and the concentration changes were controlled spectrophotometrically for $24 \mathrm{~h}$ (Supplementary Fig. S3).

Identification of the cleavage site of the substrate. The cleavage site was identified in $10 \mathrm{mM}$ $\mathrm{NH}_{4} \mathrm{HCO}_{3}$. The volume of the reaction mixture was $100 \mu \mathrm{l}$ with $20 \mu \mathrm{M}$ Abz-RSVIK(Dnp), $2.5 \mathrm{nM}$ PLN, and $7.5 \mathrm{nM}$ TLN. The mixture was incubated for 0,20 , or $40 \mathrm{~min}$ at $37^{\circ} \mathrm{C}$ and immediately frozen at $-20^{\circ} \mathrm{C}$ and lyophilized. Control samples were produced in a similar way but without the enzyme or the substrate. Dry samples were dissolved in $150 \mu \mathrm{M}$ methanol (HPLC grade, $\geq 99.9 \%$; Biosolve, Netherlands). The hydrolysis products were analyzed using an amaZon SL mass spectrometer (Bruker Daltonics, Germany) in positive ion mode. The samples were introduced directly into the ionization chamber with a flow rate of $200 \mu \mathrm{l} / \mathrm{h}$. Electrospray ionization was performed at $4000 / 500 \mathrm{~V}$ and the drying gas (nitrogen) temperature of $160^{\circ} \mathrm{C}$. The ion charge control value (ICC) was set to 200000 with a maximum accumulation time of $50 \mathrm{~ms}$. The mass measurement range in the analyzer was $100-1800 \mathrm{~m} / \mathrm{z}$. The raw data were processed with Data Analysis 4.0 (Bruker Daltoniks, Germany).

Protease activity assay using Abz-RSVIK(Dnp) and hydrolysis kinetic parameters. Desired substrate concentrations were obtained by dilution of the stock in DMSO in $50 \mathrm{mM}$ Tris- $\mathrm{HCl}$ (pH 7.4). In the activity assay, DMSO concentration did not exceed $2 \%$ in the reaction mix.

For the activity assay, $100 \mu \mathrm{l}$ of the substrate solution at $2-130 \mathrm{mM}$ in $50 \mathrm{mM}$ Tris- $\mathrm{HCl}(\mathrm{pH} 7.4)$ was added into a well of a black 96 -well plate. After incubation at $37^{\circ} \mathrm{C}$ for $10 \mathrm{~min}, 25 \mu \mathrm{l}$ of the substrate were taken away and replaced with $25 \mu \mathrm{l}$ of the enzyme solution in the same buffer preheated at $37^{\circ} \mathrm{C}$, and time-related fluorescence changes were monitored on an Infinite M200 Pro (Tecan, Switzerland). Measurements were taken at $37^{\circ} \mathrm{C}$ every $10 \mathrm{~s}$ for $2-5 \mathrm{~min}$ at the excitation and emission wavelengths of 320 and $420 \mathrm{~nm}$, respectively. Fluorescence varied linearly for $3 \mathrm{~min}$. The initial hydrolysis rates were determined from the data obtained within the first $95 \mathrm{~s}$ of the reaction. The concentrations of PLN and TLN in the reaction mix were $2.5 \mathrm{nM}$. In parallel with the hydrolysis rate measurements, the control was conducted with the buffer added to a well instead of an enzyme solution. The main goal of this control was to determine the substrate concentration in the reaction mix. After the incubation, the substrate solution was transferred into a spectrophotometer cell to measure its optical density at $365 \mathrm{~nm}$. The concentration calculated from the obtained OD was taken as the initial substrate concentration in the hydrolysis reaction.

A calibration curve was plotted to convert the obtained relative fluorescence data into the hydrolyzed substrate concentration. Abz-RSVIK(Dnp) was completely hydrolyzed by PLN after incubation of the reaction mix containing $15 \mu \mathrm{M}$ substrate and $12.5 \mathrm{nM}$ PLN in $50 \mathrm{mM}$ Tris- $\mathrm{HCl}(\mathrm{pH} 7.4)$ at $37^{\circ} \mathrm{C}$ for $15 \mathrm{~min}$. The course of the reaction was followed by fluorescence changes as described above. The hydrolysis was considered as complete when the fluorescence signal remained steady even after an extra portion of the enzyme was added (Supplementary Fig. S4). After the incubation, serial dilutions of the reaction mix were made, introduced into wells of a 96-well plate and fluorescence was measured as described above. 2,4-Dinitrophenyl was quantified spectrophotometrically in each well as described above. The resulting values were used to plot a calibration curve in coordinates of relative fluorescence units, $\mu \mathrm{M}$.

PLN and TLN remained active under the experimental conditions for $1 \mathrm{~h}$ (Supplementary Fig. S5). 
The experimental data were fit to the Michaelis-Menten equation to calculate the $\mathrm{K}_{\mathrm{M}} и \mathrm{k}_{\mathrm{cat}}$. Nonlinear regression was performed using SigmaPlot 11 (Systat Software, USA).

Protease activity assay using azocasein. A mixture of $100 \mu \mathrm{l} 1 \%$ azocasein in $50 \mathrm{mM}$ Tris- $\mathrm{HCl}(\mathrm{pH} 7.4)$ with $50 \mu \mathrm{l}$ of the enzyme in the same buffer was incubated at $37^{\circ} \mathrm{C}$ for $1 \mathrm{~h}$. The reaction was stopped by adding $200 \mu \mathrm{l}$ of $10 \%$ trichloroacetic acid. After centrifugation $(12,400 \mathrm{~g}$ for $10 \mathrm{~min}), 250 \mu \mathrm{l}$ of the supernatant was mixed with $50 \mu \mathrm{l}$ of $4 \mathrm{M} \mathrm{NaOH}$ to measure the absorbance at $450 \mathrm{~nm}$ on an Infinite M200 Pro (Tecan, Switzerland). The activity unit was defined as the amount of enzyme that changed the absorbance by one optical density unit per min.

Escherichia coli cultivation and sampling. E. coli TG1 cells transformed with pUC19 or pSP $1.8^{12}$ were grown overnight with aeration at $37^{\circ} \mathrm{C}$ in Lennox $\mathrm{LB}^{40}$ containing $100 \mu \mathrm{g} / \mathrm{ml}$ ampicillin. After measuring the culture optical densities at $600 \mathrm{~nm}\left(\mathrm{OD}_{600 \mathrm{~nm}}\right), 6 \mathrm{ml}$ of the cultures were centrifuged at $3200 \mathrm{~g}$ for $10 \mathrm{~min}$. The cell pellets were resuspended in $0.6 \mathrm{ml}$ of $50 \mathrm{mM}$ Tris- $\mathrm{HCl}(\mathrm{pH} 7.4)$ and ultrasonicated at $4{ }^{\circ} \mathrm{C}$ for $60 \mathrm{~s}$ with $30 \%$ of the duty cycle. After centrifugation $\left(8600 \mathrm{~g}, 10 \mathrm{~min}, 4^{\circ} \mathrm{C}\right)$, the supernatants were diluted with the same buffer. One $\mathrm{ml}$ of the final solution contained lysate made from $\sim 2 \cdot 10^{6}$ bacterial cells. Lysed cells were quantified at $\mathrm{OD}_{600} \mathrm{Con}_{-}$ sidering that $\mathrm{OD}_{600 \mathrm{~nm}}=1$ corresponds to $8 \cdot 10^{8}$ cells $/ \mathrm{ml}^{47}$. For the protease activity assay using Abz-RSVIK(Dnp), $25 \mu \mathrm{l}$ of each diluted solution was used.

\section{Data Availability}

All data generated or analyzed during this study are included in this published article (and its Supplementary Information Files).

\section{References}

1. Demidyuk, I. V., Gromova, T. Y. \& Kostrov, S. V. Protealysin in Handbook of Proteolytic Enzymes Vol. 1 (eds Rawlings, N.D. \& Salvesen, G.) Ch. 123, 507-602 (Academic Press, 2013).

2. Bozhokina, E., Khaitlina, S. \& Adam, T. Grimelysin, a novel metalloprotease from Serratia grimesii, is similar to ECP32. Biochem. Biophys. Res. Commun. 367, 888-892, https://doi.org/10.1016/j.bbrc.2008.01.003 (2008).

3. Tsaplina, O. A. et al. Probing for actinase activity of protealysin. Biochemistry (Mosc) 74, 648-654 (2009).

4. Bozhokina, E. S. et al. Bacterial invasion of eukaryotic cells can be mediated by actin-hydrolysing metalloproteases grimelysin and protealysin. Cell. Biol. Int. 35, 111-118, https://doi.org/10.1042/CBI20100314 (2011).

5. Tsaplina, O., Efremova, T., Demidyuk, I. \& Khaitlina, S. Filamentous actin is a substrate for protealysin, a metalloprotease of invasive Serratia proteamaculans. FEBS J. 279, 264-274, https://doi.org/10.1111/j.1742-4658.2011.08420.x (2012).

6. Tsaplina, O., Bozhokina, E., Mardanova, A. \& Khaitlina, S. Virulence factors contributing to invasive activities of Serratia grimesii and Serratia proteamaculans. Arch. Microbiol., 1-8, https://doi.org/10.1007/s00203-014-1079-7 (2015).

7. Eshwar, A. K., Wolfrum, N., Stephan, R., Fanning, S. \& Lehner, A. Interaction of matrix metalloproteinase-9 and Zpx in Cronobacter turicensis LMG 23827(T) mediated infections in the zebrafish model. Cell. Microbiol. 20, e12888, https://doi.org/10.1111/cmi.12888 (2018).

8. Cabral, C. M., Cherqui, A., Pereira, A. \& Simoes, N. Purification and characterization of two distinct metalloproteases secreted by the entomopathogenic bacterium Photorhabdus sp. strain Az29. Appl. Environ. Microbiol. 70, 3831-3838, https://doi.org/10.1128/ aem.70.7.3831-3838.2004 (2004).

9. Held, K. G., LaRock, C. N., D’Argenio, D. A., Berg, C. A. \& Collins, C. M. A metalloprotease secreted by the insect pathogen Photorhabdus luminescens induces melanization. Appl. Environ. Microbiol. 73, 7622-7628, https://doi.org/10.1128/aem.01000-07 (2007).

10. Feng, T. et al. Characterization of an extensin-modifying metalloprotease: N-terminal processing and substrate cleavage pattern of Pectobacterium carotovorum Prt1. Appl. Microbiol. Biotechnol. 98, 10077-10089, https://doi.org/10.1007/s00253-014-5877-2 (2014).

11. Kyostio, S. R., Cramer, C. L. \& Lacy, G. H. Erwinia carotovora subsp. carotovora extracellular protease: characterization and nucleotide sequence of the gene. J. Bacteriol. 173, 6537-6546 (1991).

12. Demidyuk, I. V. et al. Cloning, sequencing, expression, and characterization of protealysin, a novel neutral proteinase from Serratia proteamaculans representing a new group of thermolysin-like proteases with short N-terminal region of precursor. Protein Expr. Purif. 47, 551-561, https://doi.org/10.1016/j.pep.2005.12.005 (2006).

13. Kothary, M. H., McCardell, B. A., Frazar, C. D., Deer, D. \& Tall, B. D. Characterization of the zinc-containing metalloprotease encoded by zpx and development of a species-specific detection method for Enterobacter sakazakii. Appl. Environ. Microbiol. 73, 4142-4151 (2007).

14. Kwon, Y. T., Lee, H. H. \& Rho, H. M. Cloning, sequencing, and expression of a minor protease-encoding gene from Serratia marcescens ATCC21074. Gene 125, 75-80 (1993).

15. Zamaliutdinova, N. M., Minnullina, L. F., Sharipova, M. R. \& Mardanova, A. M. New metalloendopeptidase of Morganella morganii ZM. Bioorg. Khim. 40, 682-687 (2014).

16. Demidyuk, I. V., Gromova, T. Y. \& Kostrov, S. V. The propeptide is required for in vivo formation of active protealysin. Protein. Pept. Lett. 22, 509-513 (2015).

17. Matveyev, V. V., Usmanova, A. M., Morozova, A. V., Collins, J. H. \& Khaitlina, S. Y. Purification and characterization of the proteinase ECP 32 from Escherichia coli A2 strain. Biochim. Biophys. Acta. 1296, 55-62 (1996).

18. Feder, J. A spectrophotometric assay for neutral protease. Biochem. Biophys. Res. Commun. 32, 326-332 (1968).

19. Filippova, I., Lysogorskaia, E. N., Oksenoit, E. S., Troshchenkova, E. P. \& Stepanov, V. M. New fluorescent substrates for metalloendopeptidases with internal quenching of fluorescence. Bioorg. Khim. 14, 467-471 (1988).

20. Oda, K. et al. Exploring the subsite-structure of vimelysin and thermolysin using FRETS-libraries. FEBS Lett. 579, 5013-5018, https://doi.org/10.1016/j.febslet.2005.07.089 (2005).

21. Weimer, S., Oertel, K. \& Fuchsbauer, H. L. A quenched fluorescent dipeptide for assaying dispase- and thermolysin-like proteases. Anal. Biochem. 352, 110-119, https://doi.org/10.1016/j.ab.2006.02.029 (2006).

22. Elston, C., Wallach, J. \& Saulnier, J. New continuous and specific fluorometric assays for Pseudomonas aeruginosa elastase and LasA protease. Anal. Biochem. 368, 87-94, https://doi.org/10.1016/j.ab.2007.04.041 (2007).

23. Al-Abdullah, I. H., Bagramyan, K., Bilbao, S., Qi, M. \& Kalkum, M. Fluorogenic peptide substrate for quantification of bacterial enzyme activities. Sci. Rep. 7, 44321, https://doi.org/10.1038/srep44321 (2017).

24. Kempf, O., Kempf, K., Schobert, R. \& Bombarda, E. Hydrodabcyl: a superior hydrophilic alternative to the dark fluorescence quencher dabcyl. Anal. Chem. 89, 11893-11897, https://doi.org/10.1021/acs.analchem.7b03488 (2017). 
25. Ripp, S., Turunen, P., Minot, E. D., Rowan, A. E. \& Blank, K. G. Deciphering design principles of Förster resonance energy transferbased protease substrates: thermolysin-like protease from Geobacillus stearothermophilus as a test case. ACS Omega 3, 4148-4156, https://doi.org/10.1021/acsomega.7b02084 (2018).

26. Schechter, I. \& Berger, A. On the size of the active site in proteases. I. Papain. Biochem. Biophys. Res. Commun. 27, 157-162 (1967).

27. Demidyuk, I. V. et al. Crystal structure of the protealysin precursor: insights into propeptide function. J. Biol. Chem. 285, 2003-2013, https://doi.org/10.1074/jbc.M109.015396 (2010).

28. Khaitlina, S. et al. Physico-chemical properties of actin cleaved with bacterial protease from E. coli A2 strain. FEBS Lett 279, 49-51 (1991).

29. Gromova, T. Y., Demidyuk, I. V., Kozlovskiy, V. I., Kuranova, I. P. \& Kostrov, S. V. Processing of protealysin precursor. Biochimie 91 , 639-645, https://doi.org/10.1016/j.biochi.2009.03.008 (2009).

30. Chukhontseva, K. N., Salnikov, V. V., Morenkov, O. S., Kostrov, S. V. \& Demidyuk, I. V. Protealysin is not secreted constitutively. Protein Pept. Lett. 26, 221-226, https://doi.org/10.2174/0929866526666181212114907 (2019).

31. Demidyuk, I. V., Gasanov, E. V., Safina, D. R. \& Kostrov, S. V. Structural organization of precursors of thermolysin-like proteinases. Protein J. 27, 343-354, https://doi.org/10.1007/s10930-008-9143-2 (2008).

32. Poreba, M. \& Drag, M. Current strategies for probing substrate specificity of proteases. Curr. Med. Chem. 17, 3968-3995 (2010).

33. Poreba, M. et al. Highly sensitive and adaptable fluorescence-quenched pair discloses the substrate specificity profiles in diverse protease families. Sci. Rep. 7, 43135, https://doi.org/10.1038/srep43135 (2017).

34. Hirata, I. Y. et al. Internally quenched fluorogenic protease substrates: Solid-phase synthesis and fluorescence spectroscopy of peptides containing ortho-aminobenzoyl/dinitrophenyl groups as donor-acceptor pairs. Lett. Pept. Sci. 1, 299-308, https://doi. org/10.1007/bf00119771 (1995).

35. Kazanina, G. A., Mirgorodskaia, E. P., Mirgorodskaia, O. A. \& Khaitlina, S. ECP 32 proteinase: characteristics of the enzyme, study of specificity. Bioorg. Khim. 21, 761-766 (1995).

36. Duan, Y. \& Laursen, R. A. Protease substrate specificity mapping using membrane-bound peptides. Anal. Biochem. 216, 431-438 (1994).

37. Gron, H., Meldal, M. \& Breddam, K. Extensive comparison of the substrate preferences of two subtilisins as determined with peptide substrates which are based on the principle of intramolecular quenching. Biochemistry 31, 6011-6018 (1992).

38. Narahashi, Y. Pronase in Methods in Enzymology Vol. 19, 651-664 (Academic Press, 1970).

39. Anindyajati, Artarini, A. A., Riani, C. \& Retnoningrum, D. S. Plasmid copy number determination by quantitative polymerase chain reaction. Sci. Pharm. 84, 89-101, https://doi.org/10.3797/scipharm.ISP.2015.02 (2016).

40. Lennox, E. S. Transduction of linked genetic characters of the host by bacteriophage P1. Virology 1, 190-206 (1955).

41. Li, W. \& Godzik, A. Cd-hit: a fast program for clustering and comparing large sets of protein or nucleotide sequences. Bioinformatics 22, 1658-1659, https://doi.org/10.1093/bioinformatics/btl158 (2006)

42. Larkin, M. A. et al. Clustal W and Clustal X version 2.0. Bioinformatics 23, 2947-2948, https://doi.org/10.1093/bioinformatics/ btm404 (2007).

43. Crooks, G. E., Hon, G., Chandonia, J. M. \& Brenner, S. E. WebLogo: a sequence logo generator. Genome Res. 14, 1188-1190 (2004).

44. Bradford, M. M. A rapid and sensitive method for the quantitation of microgram quantities of protein utilizing the principle of protein-dye binding. Anal. Biochem. 72, 248-254 (1976).

45. Gasparov, V. S. \& Degtiar, V. G. Protein determination by binding with the dye Coomassie brilliant blue G-250. Biokhimiia 59, 763-777 (1994)

46. Johanning, K. et al. Specificity of prohormone convertase 2 on proenkephalin and proenkephalin-related substrates. J. Biol. Chem. 273, 22672-22680 (1998).

47. Sambrook, J., Fritsch, E. F. \& Maniatis, T. Molecular Cloning: A Laboratory Manual. (Cold Spring Harbor Laboratory, 1982).

\section{Acknowledgements}

The work was carried out using equipment of the Center of Cellular and Gene Technology of the Institute of Molecular Genetics of the Russian Academy of Sciences. This work was supported in part by the "Molecular and Cell Biology" Program of the Russian Academy of Sciences and by the Russian Foundation for Basic Research (Project Nos 19-04-00756, 18-04-00319, and 17-00-00189).

\section{Author Contributions}

M.K. and I.L. performed enzyme activity analysis, K.Ch. purified protealysin and worked with bacteria, M.P. performed mass-spectrometry experiments, S.K. and I.D. conceived and supervised the studies. The manuscript was written by M.K., M.P. and I.D. All authors participated in the discussion and reviewed the manuscript.

\section{Additional Information}

Supplementary information accompanies this paper at https://doi.org/10.1038/s41598-019-50764-2.

Competing Interests: The authors declare no competing interests.

Publisher's note Springer Nature remains neutral with regard to jurisdictional claims in published maps and institutional affiliations.

Open Access This article is licensed under a Creative Commons Attribution 4.0 International License, which permits use, sharing, adaptation, distribution and reproduction in any medium or format, as long as you give appropriate credit to the original author(s) and the source, provide a link to the Creative Commons license, and indicate if changes were made. The images or other third party material in this article are included in the article's Creative Commons license, unless indicated otherwise in a credit line to the material. If material is not included in the article's Creative Commons license and your intended use is not permitted by statutory regulation or exceeds the permitted use, you will need to obtain permission directly from the copyright holder. To view a copy of this license, visit http://creativecommons.org/licenses/by/4.0/.

(C) The Author(s) 2019 